\title{
Notes on Statistical Tests for Nonidentically Distributed Observations
}

\author{
Andrew M. Zubkov ${ }^{1}$ \\ Steklov Mathematical Institute of RAS, Moscow, Russia
}

\begin{abstract}
We discuss a problem of testing simple statistical hypotheses on the nonidentically distributed observations. A possibility of using the method Monte-Carlo for choosing the critical value in Neyman - Pearson test is pointed out. We propose also an approach permitting to reduce the problem considered to a problem of testing hypotheses on uniformity vs nonuniformity of distributions on the interval $(0,1)$.
\end{abstract}

Keywords: Hypotheses Testing, Nonhomogeneous Observations, Likelihood Ratio Ordering, Variational Distance.

\section{Introduction}

Let us consider a classical problem: we observe a sequence $\xi_{1}, \ldots, \xi_{n}$ of random variables (the structure of the set of their values is unessential) and wish to test two statistical hypotheses $\left(H_{0}\right.$ and $\left.H_{1}\right)$ on the distributions of these random variables. According to the Neyman - Pearson fundamental lemma (see Lehmann, 1986) the most powerful test in this problem should be based on the likelihood ratio

$$
L_{n}=L_{n}\left(\xi_{1}, \ldots, \xi_{n}\right)=\frac{p_{0}\left(\xi_{1}, \ldots, \xi_{n}\right)}{p_{1}\left(\xi_{1}, \ldots, \xi_{n}\right)}
$$

where $p_{0}$ and $p_{1}$ are densities (probabilities in discrete case) of the outcome $\left(\xi_{1}, \ldots, \xi_{n}\right)$. Given the probability $\alpha$ of choosing $H_{1}$ when $H_{0}$ is valid we have to find a number $c(\alpha)$ such that $\mathbf{P}\left\{L_{n} \leq c(\alpha) \mid H_{0}\right\}=\alpha$ (with possible randomization if the distribution of $L_{n}$ has an atom at $c(\alpha)$ ). If such an $c(\alpha)$ is found (and randomization is unnecessary) we may choose the hypothesis $H_{0}$ if $L_{n}>c(\alpha)$ and choose $H_{1}$ in the opposite case. This criterion has minimal probability of choosing $H_{0}$ when $H_{1}$ is valid in the set of all criteria such that the probability of choosing $H_{1}$ when $H_{0}$ is valid equals to $\alpha$.

From a practical viewpoint a serious drawback of Neyman - Pearson criterion is the necessity to compute $c(\alpha)$. This problem has an exact solution if the equation for $c(\alpha)$ is solvable. In the case of independent identically distributed observations (with known mean and variance of $\log L_{1}$ ) and large $n$ it is possible to use normal approximation for the distribution of $\log L_{n}$. But just in the simple case when $\xi_{1}, \ldots, \xi_{n}$ are independent but nonidentically distributed (for example, if $\xi_{1}, \ldots, \xi_{n}$ correspond to different characteristics of random objects) the computation of $c(\alpha)$ may become a hard problem.

This note consists of two parts. The content of the first part is an almost trivial idea to use the Monte-Carlo method to estimate $c(\alpha)$. In the second part we suggest a hint permitting to reduce the statistical problem with nonidentically distributed observations to a problem of testing hypothesis on uniformity vs nonuniformity of distributions on the interval $(0,1)$.

\footnotetext{
${ }^{1}$ This research was supported by the Leading Scientific Schools Support Fund of the President of Russia Federation, grant 1758.2003.1, and by the Program of RAS "Modern Problems of Theoretical Mathematics".
} 


\section{Monte-Carlo Estimates}

First of all note that it is possible to use Monte-Carlo approximation to $c(\alpha)$. Indeed, let us simulate $N$ independent samples $\left(\eta_{1}^{(j)}, \ldots, \eta_{n}^{(j)}\right), j=1, \ldots, N$, according to the hypothesis $H_{0}$. For each $j=1, \ldots, N$ we compute $\lambda_{j}=L_{n}\left(\eta_{1}^{(j)}, \ldots, \eta_{n}^{(j)}\right)$. These random variables are independent and identically distributed:

$$
\mathbf{P}\left\{\lambda_{j} \leq x\right\}=F_{0}(x):=\mathbf{P}\left\{L_{n} \leq x \mid H_{0}\right\} .
$$

So, $\mathbf{P}\left\{F_{0}\left(\lambda_{j}\right)<y\right\} \leq y$ for all $y \in[0,1]$, and $\mathbf{P}\left\{F_{0}\left(\lambda_{j}\right)<y\right\}=y$ if $F_{0}^{-1}(y):=$ $\sup \left\{x: F_{0}(x)<y\right\}$ is a continuity point of $F_{0}$. For large $N$ the empirical distribution function of $\lambda_{1}, \ldots, \lambda_{N}$ will approximate the distribution function $F_{0}(x)$. For example, let $\lambda_{1: N} \leq \lambda_{2: N} \leq \ldots \leq \lambda_{N: N}$ be the order statistics of $\lambda_{1}, \ldots, \lambda_{N}$. If we choose $\lambda_{[\alpha N]: N}([x]$ denotes an integer part of $x$ ) as an approximation to $c(\alpha)$ then

$$
\begin{aligned}
& \mathbf{P}\left\{F_{0}\left(\lambda_{[\alpha N]: N}\right)<\alpha-\varepsilon\right\}=\mathbf{P}\left\{\sum_{j=1}^{N} I\left(F_{0}\left(\lambda_{j}\right)<\alpha-\varepsilon\right) \geq[\alpha N]\right\} \leq \mathbf{P}\left\{\beta_{N, \alpha-\varepsilon} \geq \alpha N\right\}, \\
& \mathbf{P}\left\{F_{0}\left(\lambda_{[\alpha N]: N}\right)>\alpha+\varepsilon\right\}=\mathbf{P}\left\{\sum_{j=1}^{N} I\left(F_{0}\left(\lambda_{j}\right) \leq \alpha+\varepsilon\right)<[\alpha N]\right\} \leq \mathbf{P}\left\{\beta_{N, \alpha+\varepsilon}<\alpha N\right\},
\end{aligned}
$$

where $\beta_{N, p}$ denotes random variable having the binomial distribution with parameters $(N, p)$. These equations may be used to estimate the accuracy of approximation of $c(\alpha)$ as a function of $\alpha$ and $N$.

The same idea of using the Monte-Carlo method may be applied not before, but after observing the sequence $\xi_{1}, \ldots, \xi_{n}$. Let us simulate $N$ independent samples

$$
\left(\eta_{1}^{(j)}, \ldots, \eta_{n}^{(j)}\right), \quad j=1, \ldots, N
$$

according to the hypothesis $H_{0}$ and compute the number $\nu_{N}$ of $j=1, \ldots, N$ such that $\lambda_{j} \leq L_{n}\left(\xi_{1}, \ldots, \xi_{n}\right)$. Then the value $\frac{\nu_{N}}{N}$ is a consistent (as $\left.N \rightarrow \infty\right)$ statistical estimate of $F_{0}\left(L_{n}\left(\xi_{1}, \ldots, \xi_{n}\right)\right)$. So the inequality $\frac{\nu_{N}}{N} \geq \alpha$ is asymptotically (as $N \rightarrow \infty$ ) equivalent to the Neyman - Pearson criterion. It may be shown that

$$
-\max _{0 \leq y \leq 1} \mathbf{P}\left\{F_{0}\left(L_{n}\right)=y\right\} \leq \mathbf{P}\left\{\nu_{N} \leq m\right\}-\frac{m+1}{N+1} \leq 0, \quad m=1, \ldots, N .
$$

Computation of estimates $\frac{\nu_{N}}{N}$ for each observation of $\xi_{1}, \ldots, \xi_{n}$ takes more time but it rules out inevitable systematic bias appearing when a single Monte-Carlo estimate of $c(\alpha)$ is used.

\section{Reduction to a Simpler Problem}

Consider another possibility to test hypotheses $H_{0}$ and $H_{1}$ on independent nonidentically distributed random variables $\xi_{1}, \ldots, \xi_{n}$.

We begin with the case when for each $j=1, \ldots, N$ the distributions of $\xi_{j}$ under both $H_{0}$ and $H_{1}$ are absolutely continuous with common support. Let $p_{i, j}(x)$ be a density of $\xi_{j}$ 
under hypothesis $H_{i}, i=0,1, j=1, \ldots, N$. Denote $r_{j}(x)=\frac{p_{0, j}(x)}{p_{1, j}(x)}, j=1, \ldots, N$, and introduce distribution functions

$$
G_{j}(x)=\mathbf{P}\left\{r_{j}\left(\xi_{j}\right) \leq x \mid H_{0}\right\}=\int_{u: r_{j}(u) \leq x} p_{0, j}(u) d u, j=1, \ldots, N .
$$

Definition of $G_{j}(x)$ seems complex, but it may be simplified in concrete cases. For example, if $r_{j}(x)$ is monotonically decreasing and $r_{j}^{-1}(y)=\sup \left\{x: r_{j}(x) \geq y\right\}$ then

$$
G_{j}(x)=\int_{r_{j}^{-1}(x)}^{\infty} p_{0, j}(u) d u, j=1, \ldots, N
$$

if $r_{j}(x)$ is unimodal (i.e. $r_{j}(x)$ is increasing from $r_{j}(-\infty)$ to $r_{j}\left(a_{j}\right)$ on $\left[-\infty, a_{j}\right]$ and decreasing from $r_{j}\left(a_{j}\right)$ to $r_{j}(\infty)$ on $\left.\left[a_{j}, \infty\right)\right)$ and

$$
\begin{gathered}
r_{j}^{+}(y)=\left\{\sup \left\{x: r_{j}(x) \geq y\right\}, \quad r_{j}(\infty)<y \leq r_{j}\left(a_{j}\right), \infty, \quad r_{j}(0) \leq y \leq r_{j}(\infty),\right. \\
r_{j}^{-}(y)=\left\{\inf \left\{x: r_{j}(x) \geq y\right\}, \quad r_{j}(0) \leq y \leq r_{j}\left(a_{j}\right),-\infty, \quad r_{j}(\infty)<y \leq r_{j}\left(a_{j}\right),\right.
\end{gathered}
$$

then

$$
G_{j}(x)=1-\int_{r_{j}^{-}(x)}^{r_{j}^{+}(x)} p_{0, j}(u) d u, \quad \min \left\{r_{j}(0), r_{j}(\infty)\right\} \leq x \leq r_{j}\left(a_{j}\right), \quad j=1, \ldots, N .
$$

Let $\zeta_{j}=G_{j}\left(r_{j}\left(\xi_{j}\right)\right), j=1, \ldots, N$. If distributions of $\xi_{1}, \ldots, \xi_{n}$ satisfy the hypothesis $H_{0}$ then random variables $\zeta_{1}, \ldots, \zeta_{N}$ are independent and uniformly distributed on $[0,1]$ :

$$
\begin{gathered}
Z_{0}(x):=\mathbf{P}\left\{\zeta_{j} \leq x \mid H_{0}\right\}=\mathbf{P}\left\{G_{j}\left(r_{j}\left(\xi_{j}\right)\right) \leq x \mid H_{0}\right\}= \\
=\mathbf{P}\left\{r_{j}\left(\xi_{j}\right) \leq G_{j}^{-1}(x) \mid H_{0}\right\}=G_{j}\left(G_{j}^{-1}(x)\right)=x, \quad x \in[0,1], j=1, \ldots, N,
\end{gathered}
$$

where $G^{-1}$ denotes inverse function for $G$.

If hypothesis $H_{1}$ is valid then random variables $\zeta_{1}, \ldots, \zeta_{N}$ are independent and have nonuniform distributions. The distribution function of $\zeta_{j}$ under the hypothesis $H_{1}$ takes the form

$$
Z_{1, j}(x):=\mathbf{P}\left\{\zeta_{j} \leq x \mid H_{1}\right\}=\mathbf{P}\left\{G_{j}\left(r_{j}\left(\xi_{j}\right)\right) \leq x \mid H_{1}\right\}=\mathbf{P}\left\{r_{j}\left(\xi_{j}\right) \leq G_{j}^{-1}(x) \mid H_{1}\right\} .
$$

Theorem 1. For any $j=1, \ldots, N$ the function $Z_{1, j}(x)$ is concave on $[0,1]$ and $\rho_{j}=\max _{0 \leq x \leq 1}\left(Z_{1, j}(x)-Z_{0}(x)\right)$ is equal to the variational distance between distributions of $\xi_{j}$ under hypotheses $H_{0}$ and $H_{1}$. Further,

$$
\begin{gathered}
\mathbf{E}\left\{\zeta_{j} \mid H_{0}\right\}=\frac{1}{2}, \quad \mathbf{E}\left\{\zeta_{j} \mid H_{1}\right\} \leq \frac{1-\rho_{j}}{2}, \\
\mathbf{D}\left\{\zeta_{j} \mid H_{0}\right\}=\frac{1}{12}, \quad \mathbf{D}\left\{\zeta_{j} \mid H_{1}\right\} \leq \frac{1}{4} .
\end{gathered}
$$


Corollary. We have $Z_{1, j}(x)>Z_{0}(x)=x$ for all $x \in(0,1)$ and all $j=1, \ldots, N$.

PROOF. Let

$$
G_{1, j}(x)=\mathbf{P}\left\{r_{j}\left(\xi_{j}\right) \leq x \mid H_{1}\right\}=\int_{u: r_{j}(u) \leq x} p_{1, j}(u) d u, j=1, \ldots, N .
$$

Then $Z_{1, j}(x)=G_{1, j}\left(G_{j}^{-1}(x)\right)$. So,

$$
\begin{gathered}
\frac{d}{d x} Z_{1, j}(x)=\frac{d}{d x} G_{1, j}\left(G_{j}^{-1}(x)\right)=\left.\frac{d}{d v} G_{1, j}(v)\right|_{v=G_{j}^{-1}(x)} \frac{d}{d x} G_{j}^{-1}(x)= \\
=\frac{\left.\frac{d}{d v} G_{1, j}(v)\right|_{v=G_{j}^{-1}(x)}}{\left.\frac{d}{d v} G_{j}(v)\right|_{v=G_{j}^{-1}(x)}}=\frac{\lim _{\Delta \downarrow 0} \frac{1}{\Delta} \lim _{u \downarrow G_{j}^{-1}(x)-\Delta \leq r_{j}(u) \leq G_{j}^{-1}(x)} \frac{1}{\Delta} p_{u: j}(u) d u}{\int_{u: G_{j}^{-1}(x)-\Delta \leq r_{j}(u) \leq G_{j}^{-1}(x)} p_{0, j}(u) d u}=\frac{1}{G_{j}^{-1}(x)}
\end{gathered}
$$

is nonincreasing, i.e. $Z_{1, j}(x)$ is concave.

Note that distribution functions $Z_{0}(x)=x, Z_{1, j}(x), x \in[0,1]$, are absolutely continuous with densities $z_{0}(x)=1, z_{1, j}(x)=\frac{1}{G_{j}^{-1}(x)}, x \in[0,1]$, and satisfy conditions $Z_{0}(0)=Z_{1, j}(0)=0, Z_{0}(1)=Z_{1, j}(1)=1$. Let $c_{j}=\sup \left\{x \in[0,1]: z_{1, j}(x) \geq 1\right\}$. Due to monotonicity of $z_{1, j}(x)$ we have

$$
\begin{gathered}
\rho_{j}=\max _{0 \leq x \leq 1}\left(Z_{1, j}(x)-Z_{0}(x)\right)=Z_{1, j}\left(c_{j}\right)-Z_{0}\left(c_{j}\right)= \\
=\frac{1}{2}\left(\int_{0}^{c_{j}}\left(z_{1, j}(x)-1\right) d x+\int_{c_{j}}^{1}\left(1-z_{1, j}(x)\right) d x\right)=\frac{1}{2} \int_{0}^{1}\left|z_{1, j}(x)-1\right| d x .
\end{gathered}
$$

Further, taking into account (5) and applying change of variable $x=G_{j}(y)$ we find

$$
\begin{gathered}
\int_{0}^{1}\left|z_{1, j}(x)-1\right| d x=\int_{0}^{1}\left|\frac{1}{G_{j}^{-1}(x)}-1\right| d x=\int_{0}^{1}\left|\frac{\left.\frac{d}{d v} G_{1, j}(v)\right|_{v=G_{j}^{-1}(x)}}{\left.\frac{d}{d v} G_{j}(v)\right|_{v=G_{j}^{-1}(x)}}-1\right| d x= \\
=\int_{-\infty}^{\infty}\left|\frac{\frac{d}{d y} G_{1, j}(y)}{\frac{d}{d y} G_{j}(y)}-1\right| d G_{j}(y)=\int_{-\infty}^{\infty}\left|\frac{d}{d y} G_{1, j}(y)-\frac{d}{d y} G_{j}(y)\right| d y= \\
=\int_{-\infty}^{\infty}\left|\frac{d}{d y} \int_{u: r_{j}(u) \leq y} p_{1, j}(u) d u-\frac{d}{d y} \int_{u: r_{j}(u) \leq y} p_{0, j}(u) d u\right| d y=\int\left|p_{1, j}(u)-p_{0, j}(u)\right| d u,
\end{gathered}
$$

i.e., $\rho_{j}$ equals to the variational distance between distributions of $\xi_{j}$ under hypotheses $H_{0}$ and $H_{1}$.

Random variable $\zeta_{j}$ under hypothesis $H_{0}$ is uniformly distributed on $[0,1]$, so

$$
\mathbf{E}\left\{\zeta_{j} \mid H_{0}\right\}=\frac{1}{2}, \quad \mathbf{D}\left\{\zeta_{j} \mid H_{0}\right\}=\frac{1}{12} .
$$


To estimate $\mathbf{E}\left\{\zeta_{j} \mid H_{1}\right\}$ we introduce piecewise linear function $L_{j}(x)$ : the graph of $L_{j}(x)$ connects points $(0,0),\left(c_{j}, Z_{1, j}\left(c_{j}\right)\right),(1,1)$. Due to the concavity of $Z_{1, j}(x)$ we have $Z_{1, j}(x) \geq L_{j}(x)$ for all $x \in[0,1]$. Now

$$
\begin{gathered}
\mathbf{E}\left\{\zeta_{j} \mid H_{1}\right\}=\int_{0}^{1}\left(1-Z_{1, j}(x)\right) d x \leq \int_{0}^{1}\left(1-L_{j}(x)\right) d x= \\
=\int_{0}^{1}(1-x) d x-\int_{0}^{1}\left(L_{j}(x)-x\right) d x=\frac{1}{2}-\frac{\rho_{j}}{2}
\end{gathered}
$$

because the area of triangle formed by graphs of $L_{j}(x)$ and $Z_{0}(x)=x$ equals to

$$
\frac{1}{2}\left(Z_{1, j}\left(c_{j}\right)-c_{j}\right)=\frac{\rho_{j}}{2}
$$

Inequality $\mathbf{D}\left\{\zeta_{j} \mid H_{1}\right\} \leq \frac{1}{4}$ is valid for any random variable with values in $[0,1]$. The theorem is proved.

Now consider the case when distributions of $\xi_{j}$ under both $H_{0}$ and $H_{1}$ are discrete with common support $T_{j}, j=1, \ldots, N$. Let

$$
P_{i, j}(t)=\mathbf{P}\left\{\xi_{j}=t \mid H_{i}\right\}, \quad t \in T_{j}=\left\{t_{j, 1}, t_{j, 2}, \ldots\right\}, \quad j=1, \ldots, N,
$$

be the distribution of $\xi_{j}$ under hypothesis $H_{i}, i=0,1$. The sets $T_{1}, \ldots, T_{N}$ are at most countable. Let $R_{j}(t)=\frac{P_{0, j}(t)}{P_{1, j}(t)}, t \in T_{j}$; the sets $R_{j}$ of values $R_{j}(t), t \in T_{j}$, are at most countable also.

It is convenient to introduce right-continuous and left-continuous distribution functions for each $j=1, \ldots, N$ :

$$
\begin{aligned}
& S_{0, j}(x)=\mathbf{P}\left\{R_{j}\left(\xi_{j}\right) \leq x \mid H_{0}\right\}=\sum_{t \in T_{j}: R_{j}(t) \leq x} P_{0, j}(t), \\
& S_{0, j}^{-}(x)=\mathbf{P}\left\{R_{j}\left(\xi_{j}\right)<x \mid H_{0}\right\}=\sum_{t \in T_{j}: R_{j}(t)<x} P_{0, j}(t), \\
& S_{1, j}(x)=\mathbf{P}\left\{R_{j}\left(\xi_{j}\right) \leq x \mid H_{1}\right\}=\sum_{t \in T_{j}: R_{j}(t) \leq x} P_{1, j}(t), \\
& S_{1, j}^{-}(x)=\mathbf{P}\left\{R_{j}\left(\xi_{j}\right)<x \mid H_{1}\right\}=\sum_{t \in T_{j}: R_{j}(t)<x} P_{1, j}(t),
\end{aligned}
$$

here $x \in[0, \infty]$. Let $S_{j}$ be a set of points with coordinates $\left(S_{0, j}(r), S_{1, j}(r)\right), r \in R_{j}$.

Lemma. For each $j=1, \ldots, N$ the set $S_{j}$ is lying on a concave curve.

PROOF. It is sufficient to prove that for any $r_{1}<r_{2}<r_{3}\left(r_{1}, r_{2}, r_{3} \in R_{j}\right)$ the slope of a chord $\left[\left(S_{0, j}\left(r_{1}\right), S_{1, j}\left(r_{1}\right)\right),\left(S_{0, j}\left(r_{2}\right), S_{1, j}\left(r_{2}\right)\right)\right]$ is greater than the slope of a chord $\left[\left(S_{0, j}\left(r_{2}\right), S_{1, j}\left(r_{2}\right)\right),\left(S_{0, j}\left(r_{3}\right), S_{1, j}\left(r_{3}\right)\right)\right]$, i.e. that

$$
\frac{S_{1, j}\left(r_{2}\right)-S_{1, j}\left(r_{1}\right)}{S_{0, j}\left(r_{2}\right)-S_{0, j}\left(r_{1}\right)}>\frac{S_{1, j}\left(r_{3}\right)-S_{1, j}\left(r_{2}\right)}{S_{0, j}\left(r_{3}\right)-S_{0, j}\left(r_{2}\right)} .
$$


But according to definitions (6) - (9) we have

$$
\begin{gathered}
\frac{S_{1, j}\left(r_{2}\right)-S_{1, j}\left(r_{1}\right)}{S_{0, j}\left(r_{2}\right)-S_{0, j}\left(r_{1}\right)}=\frac{\sum_{t \in T_{j}: r_{1}<R_{j}(t) \leq r_{2}} P_{1, j}(t)}{\sum_{t \in T_{j}: r_{1}<R_{j}(t) \leq r_{2}} P_{0, j}(t)} \geq \frac{1}{r_{2}}> \\
>\frac{\sum_{t \in T_{j}: r_{2}<R_{j}(t) \leq r_{3}} P_{1, j}(t)}{\sum_{t \in T_{j}: r_{2}<R_{j}(t) \leq r_{3}} P_{0, j}(t)}=\frac{S_{1, j}\left(r_{3}\right)-S_{1, j}\left(r_{2}\right)}{S_{0, j}\left(r_{3}\right)-S_{0, j}\left(r_{2}\right)},
\end{gathered}
$$

and Lemma is proved.

The proof of Lemma is applicable to the absolutely continuous case also, but in that case we have used an explicit formula for the density of $Z_{1, j}(x)$. To get a full analogy with the absolutely continuous case we define functions $Z_{1, j}^{*}(x), x \in[0,1], j=1, \ldots, N$, such that their graphs are convex hulls of corresponding sets $S_{j}$. In other words, these functions are continuous, satisfy conditions

$$
Z_{1, j}^{*}\left(S_{0, j}(r)\right)=S_{1, j}(r), \quad r \in R_{j}, \quad Z_{1, j}^{*}(0)=0, \quad Z_{1, j}^{*}(1)=1,
$$

and are piecewise linear on intervals $\left(S_{0, j}^{-}(r), S_{0, j}(r)\right), r \in R_{j}$, between points of the set $\bar{S}_{j}=\{0\} \cup\left\{S_{0, j}(r), r \in R_{j}\right\} \cup\{1\}$. Evidently, $Z_{1, j}^{*}$ for each $j=1, \ldots, N$ is a distribution function of the mixture of uniform distributions on intervals $\left(S_{0, j}^{-}(r), S_{0, j}(r)\right), r \in \bar{S}_{j}$, with weights $S_{1, j}(r)-S_{1, j}^{-}(r)$.

It is obvious that mixture of uniform distributions on intervals $\left(S_{0, j}^{-}(r), S_{0, j}(r)\right), r \in$ $\bar{S}_{j}$, with weights $S_{0, j}(r)-S_{0, j}^{-}(r)$ is a uniform distribution on $[0,1]$ and has distribution function $Z_{0, j}^{*}(x)=x, x \in[0,1]$.

Now to define randomized statistics $\zeta_{j}=\zeta_{j}\left(\xi_{j}\right)$ we introduce auxiliary random variables $\alpha_{1}, \ldots, \alpha_{N}$ uniformly distributed on an interval $[0,1]$ and put

$$
\zeta_{j}=\alpha_{j} S_{0, j}^{-}(r)+\left(1-\alpha_{j}\right) S_{0, j}(r) \quad \text { if } \quad R_{j}\left(\xi_{j}\right)=r .
$$

Being defined in such a way random variable $\zeta_{j}$ is uniformly distributed on $[0,1]$ under hypothesis $H_{0}$ and has distribution function $Z_{1, j}^{*}(x)$ under hypothesis $H_{1}$.

Theorem 2. For any $j=1, \ldots, N$ the function $Z_{1, j}^{*}(x)$ is concave on $[0,1]$ and $\rho_{j}=\max _{0 \leq x \leq 1}\left(Z_{1, j}^{*}(x)-Z_{0, j}^{*}(x)\right)$ is equal to the variational distance between distributions of $\xi_{j}$ under hypotheses $H_{0}$ and $H_{1}$. Further,

$$
\begin{gathered}
\mathbf{E}\left\{\zeta_{j} \mid H_{0}\right\}=\frac{1}{2}, \quad \mathbf{E}\left\{\zeta_{j} \mid H_{1}\right\} \leq \frac{1-\rho_{j}}{2}, \\
\mathbf{D}\left\{\zeta_{j} \mid H_{0}\right\}=\frac{1}{12}, \quad \mathbf{D}\left\{\zeta_{j} \mid H_{1}\right\} \leq \frac{1}{4} .
\end{gathered}
$$

Proof. Concavity of the function $Z_{1, j}^{*}(x)$ follows from Lemma. The function $Z_{1, j}^{*}(x)$ is linear on intervals $\left(S_{0, j}^{-}(r), S_{0, j}(r)\right), r \in R_{j}$, so $\max _{0 \leq x \leq 1}\left(Z_{1, j}^{*}(x)-Z_{0, j}^{*}(x)\right)$ is attained on the closure of the set $\{0\} \cup\left\{S_{0, j}(r), r \in R_{j}\right\}$. Further, for any $r \in R_{j}$

$$
Z_{1, j}^{*}\left(S_{0, j}(r)\right)-Z_{0, j}^{*}\left(S_{0, j}(r)\right)=S_{1, j}(r)-S_{0, j}(r)=
$$




$$
=\sum_{t \in T_{j}: R_{j}(t) \leq r} P_{1, j}(t)-\sum_{t \in T_{j}: R_{j}(t) \leq r} P_{0, j}(t)=\sum_{t \in T_{j}: P_{0, j}(t) \leq r P_{1, j}(t)}\left(P_{1, j}(t)-P_{0, j}(t)\right) .
$$

The summands in the last sum are positive for $r<1$ and negative for $r>1$; it follows that $\max _{0 \leq x \leq 1}\left(Z_{1, j}^{*}(x)-Z_{0, j}^{*}(x)\right)$ is attained at the point $x=\sup \left\{S_{0, j}(r): r \leq 1\right\}$ and is equal to

$$
\frac{1}{2} \sum_{t \in T_{j}}\left|P_{1, j}(t)-P_{0, j}(t)\right|,
$$

i.e. to the variational distance between distributions of $\xi_{j}$ under hypotheses $H_{0}$ and $H_{1}$.

Moments of $\zeta_{j}$ are estimated as in Theorem 1. Theorem 2 is proved.

Theorems 1 and 2 reduce the problem of testing two simple statistical hypotheses on the distribution of independent nonidentically distributed random variables $\xi_{1}, \ldots, \xi_{N}$ (taking values of any nature) to the problem of testing hypothesis $H_{0}^{\prime}$ : independent random variables $\zeta_{1}\left(\xi_{1}\right), \ldots, \zeta_{N}\left(\xi_{n}\right)$ are uniformly distributed on $[0,1]$ against hypothesis $H_{1}^{\prime}$ : independent random variables $\zeta_{1}\left(\xi_{1}\right), \ldots, \zeta_{N}\left(\xi_{n}\right)$ taking values in $[0,1]$ have concave nonuniform distribution functions $Z_{1, j}(x), j=1, \ldots, N$. So under $H_{0}^{\prime}$ random variables $\zeta_{1}\left(\xi_{1}\right), \ldots, \zeta_{N}\left(\xi_{n}\right)$ are identically distributed and under $H_{1}^{\prime}$ random variables $\zeta_{1}\left(\xi_{1}\right), \ldots, \zeta_{N}\left(\xi_{n}\right)$ have biases of the same sign compared to their distributions under $H_{0}^{\prime}$.

The simplest way to test $H_{0}^{\prime}$ against $H_{1}^{\prime}$ by means of these biases is to use statistics $V_{N}=\sum_{j=1}^{N} \zeta_{j}$. If $H_{0}$ is valid then $V_{N}$ is the sum of $N$ independent random variables uniformly distributed on the interval $[0,1]$, in particular,

$$
\mathbf{E}\left\{V_{N} \mid H_{0}\right\}=\frac{N}{2}, \quad \mathbf{D}\left\{V_{N} \mid H_{0}\right\}=\frac{N}{12}
$$

If $H_{1}$ is valid then $V_{N}$ is the sum of $N$ independent random variables which are stochastically smaller than random variables uniformly distributed on the interval $[0,1]$, and

$$
\mathbf{E}\left\{V_{N} \mid H_{1}\right\} \leq \sum_{j=1}^{N} \frac{1-\rho_{j}}{2}, \quad \mathbf{D}\left\{V_{N} \mid H_{1}\right\}=\sum_{j=1}^{N} \mathbf{D}\left\{\zeta_{j} \mid H_{1}\right\} \leq \frac{N}{4} .
$$

For large $N$ normal approximations may be used to estimate critical levels and error probabilities (especially if explicit formulas for $\mathbf{E}\left\{V_{N} \mid H_{1}\right\}$ and $\mathbf{D}\left\{V_{N} \mid H_{1}\right\}$ are known). But this approach, of course, is not the best possible.

Another way is to compute statistics of goodness-of-fit criteria (for example, Kolmogorov - Smirnov statistics) for $H_{0}^{\prime}$ and $H_{1}^{\prime}$ separately and compare their values.

However it seems that the problem of testing $H_{0}^{\prime}$ against $H_{1}^{\prime}$ is far from being completely solved.

\section{References}

E.L. Lehmann. Testing Statistical Hypotheses. Springer-Verlag, New York e.a., 2nd edition, 1986. 
Author's address:

Dr. Andrew M. Zubkov

Department of Discrete Mathematics

Steklov Mathematical Institute

Gubkina Str. 8

119991 Moscow

Russia

Tel. +7095 1351519

Fax +70951350555

E-mail: zubkov@mi.ras.ru 